\title{
The Impact of Excluding Patients with End-Stage Knee Disease in Intra-Articular Hyaluronic Acid Trials: A Systematic Review and Meta-Analysis
}

\author{
Mathew Nicholls (D) - Peter Shaw · Faizan Niazi · Mohit Bhandari • \\ Asheesh Bedi
}

Received: October 1, 2018 / Published online: December 1, 2018

(C) The Author(s) 2018

\begin{abstract}
Introduction: The Kellgren-Lawrence (K-L) grade is the most commonly used measure of radiographic disease severity in knee osteoarthritis (OA). Studies suggest that intraarticular hyaluronic acid (IA-HA) should only be considered in cases of early stage knee OA. The purpose of this review was to determine if trials administering IA-HA in early-moderate knee OA patients demonstrated greater pain relief than studies that also included patients with endstage disease.
\end{abstract}

Enhanced Digital Features To view enhanced digital features for this article go to https://doi.org/10.6084/ m9.figshare.7370192.

Electronic supplementary material The online version of this article (https://doi.org/10.1007/s12325018-0847-1) contains supplementary material, which is available to authorized users.

M. Nicholls ( $\square)$

Virginia Mason Orthopaedics and Sports Medicine, Seattle, WA, USA

e-mail: matn@mindspring.com

P. Shaw $\cdot$ F. Niazi

Ferring Pharmaceuticals Inc., Parsippany, NJ, USA

M. Bhandari

Division of Orthopaedic Surgery, McMaster

University, Hamilton, ON, Canada

A. Bedi

Department of Orthopaedic Surgery, University of

Michigan, Ann Arbor, MI, USA
Methods: We conducted a systematic search of the literature to identify randomized controlled trials (RCT) comparing IA-HA with saline injections and that diagnosed disease severity using the $\mathrm{K}-\mathrm{L}$ grade criteria. The primary outcome was mean change in pain from baseline at 4-13 weeks and 22-27 weeks. Safety was evaluated on the total number of participants experiencing a treatment-related adverse event (AE). Results: Twenty RCTs were included. In the early-moderate OA subgroup, the mean change in pain scores was statistically significant favoring IA-HA from baseline to 4-13 weeks $[\mathrm{SMD}=-0.30,95 \% \quad \mathrm{CI}-0.44$ to -0.15 , $p<0.0001]$ and within 22-27 weeks $[\mathrm{SMD}=-0.27,95 \%$ CI -0.39 to -0.16 , $p<0.00001]$. No significant differences were observed in the late OA subgroup. IA-HA was associated with a significantly greater risk of treatment-related AEs relative to saline in the late OA subgroup $[\mathrm{RR}=1.76,95 \%$ CI $1.16-2.67$, $p=0.008]$.

Conclusion: IA-HA provides significant pain relief compared to saline for patients with earlymoderate knee OA, compared to cohorts including patients with end-stage OA (KL grade $4)$, with no increase in the risk of treatmentrelated AEs, up to 6 months. Patients with endstage disease had lower levels of pain relief and may be diluting study results if included in the treatment cohort.

Funding: Ferring Pharmaceuticals. 
Keywords: Intra-articular hyaluronic acid; Knee; Osteoarthritis; Rheumatology

\section{INTRODUCTION}

Osteoarthritis (OA) is the most common joint disorder in the USA, estimated to affect over 30 million adults, and the prevalence is expected to increase for the foreseeable future [1, 2]. Joint pain is a common complaint among individuals with OA and a primary reason for them to seek medical care $[2,3]$. As the disease is chronic and nonfatal, identifying effective measures to not only prevent but also treat it will have significant impacts at both the clinical and socioeconomic levels $[1,4]$. Osteoarthritis may occur in any joint, but knee $\mathrm{OA}$ is one of the most common manifestations of this disease and, as the knee takes on a substantial amount of the weight-bearing load, can lead to significant disability if left untreated [1,4].

While surgical intervention is typically reserved for the most severe OA, more conservative therapies are initiated earlier in the disease process in effort to alleviate symptoms and delay progression. Treatment with intra-articular hyaluronic acid (IA-HA) injections is one of these options, although there is inconsistency in its recommendations across knee OA guidelines [4-7]. HA is a naturally occurring substance present in synovial fluid and the quality and quantity of HA are reduced in arthritic knees [3, 8]. IA-HA injections are typically indicated for patients who are non-responders to nonpharmacological and pharmacological therapies or experience adverse effects from these treatments $[3,8,9]$. In addition to restoring the viscoelasticity of the synovial fluid, IA-HA may also have anti-inflammatory and antinociceptive properties, and stimulate in vivo high molecular weight (HMW) HA synthesis [8]. Both basic science studies and clinical trials have demonstrated the potential benefit of HA injections [4]. While a number of different HA products are available in various injection regimens, literature has demonstrated there are also significant differences in molecular and rheological properties $[4,8,10,11]$.
Radiographs play an important role in the diagnosis of OA $[1,2]$. In clinical trials investigating knee OA, disease severity is most commonly assessed by the Kellgren-Lawrence (KL) criteria $[1,2]$. This system grades $\mathrm{OA}$ into five categories of severity, from 0 to 4 , with lower grades representing greater joint space and less disease severity [12]. Most IA-HA studies have focused on its use in patients with early to moderate knee OA (K-L grade $\leq 3)$, suggesting that HA injections should only be considered earlier in the disease process and that studies that have included patients with end-stage disease (K-L grade 4 ) have contributed to the current controversy surrounding their use $[4,13]$. A number of prior studies have demonstrated a greater response to HA therapy when baseline radiographic presentation was less severe [14-16].

The purpose of this review was to determine if the inclusion of end-stage OA (KL grade 4) participants reduced the measured effectiveness of IA-HA in randomized controlled trials measuring its effect on knee OA pain.

\section{METHODS}

\section{Literature Search}

A comprehensive literature search for relevant articles was conducted using a detailed search of the MEDLINE, EMBASE, and PubMed databases (Supplementary Material). The inclusion criteria were (1) blinded randomized controlled trial (RCT) comparing IA-HA with intra-articular saline injections; (2) knee pain or treatmentrelated adverse events (AEs) were a reported outcome; (3) described disease severity using the K-L grade criteria; and (4) articles that were published in English. After the list of eligible studies was finalized, a manual search of relevant reference lists was conducted to ensure that no potentially eligible trials were missed. This article is based on previously conducted studies and does not contain any studies with human participants or animals performed by any of the authors. 


\section{Search Results}

The literature searched identified 2198 citations (Fig. 1). After titles and abstracts were screened, 166 were included for full-text review. Nineteen studies met the predefined inclusion criteria $[14,17-34]$ and an additional study was handselected [35], for a total of 20 trials; however, one of these studies was only included in the analysis of treatment-related adverse events [29]. The hand-selected study was retrieved from the reference list of previously published articles regarding IA-HA use for knee osteoarthritis.

\section{Data Extraction and Outcome Measures}

Data extraction consisted of study characteristics, patient demographics, and reported outcome and safety measures. The primary outcome measure was the mean change in knee pain score from baseline at two separate visit windows: (1) 4-13 weeks (earlier visit) and (2) 22-27 weeks (later visit). The Western Ontario and McMaster Universities Arthritis Index (WOMAC) pain scores were extracted whenever reported. If the WOMAC pain scores were not reported, an a priori hierarchy of outcomes was used to extract the next most relevant pain measure. The hierarchy used was taken from a previous meta-analysis, and was as follows:

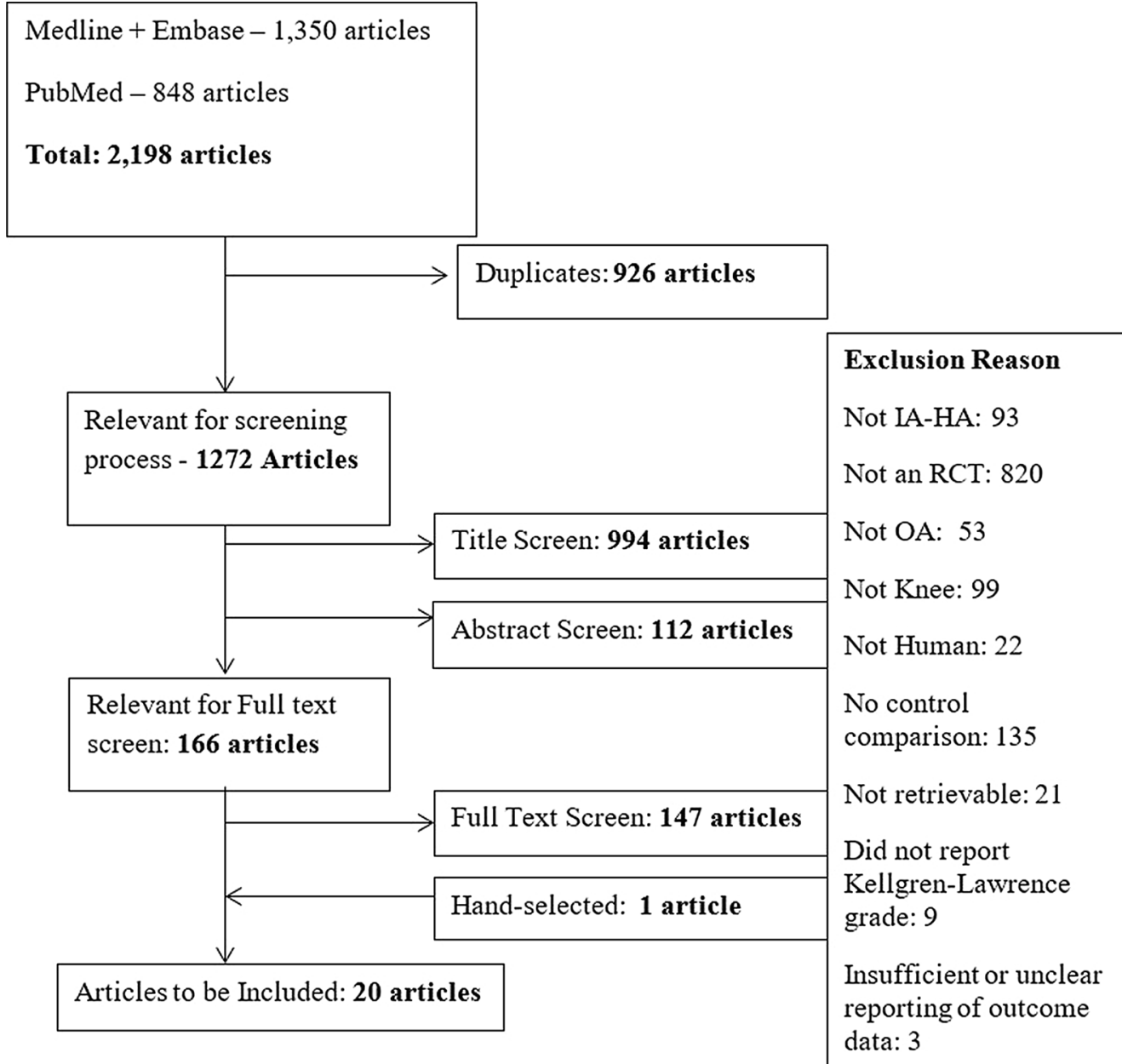

Fig. 1 Flow diagram of search results 
WOMAC pain, visual analog scale (VAS) pain on activity/walking, VAS pain on weight-bearing, VAS pain at rest, or other pain outcomes (Knee Injury and Osteoarthritis Outcome Score (KOOS), Musculoskeletal Outcomes Data Evaluation and Management System (MODEMS), Index of Severity for Osteoarthritis for the Knee (ISK) assessment, and WOMAC total score [11]. If data for these outcomes were not reported in a given study, it was not included in the primary outcome. Safety data was also extracted when possible on the total number of participants experiencing a treatment-related AE. If data for these outcomes were not reported in a given study, it was not included in the safety analysis. Data from the intent-to-treat population was used whenever possible. Data extraction was completed in duplicate by two independent reviewers.

\section{Data Analysis}

Standardized mean differences (SMD) and relative risks (RRs) were analyzed using the Cochrane Review Manager 5.3 software [36]. For continuous outcomes, a negative SMD represented a result favoring IA-HA, while a positive effect estimate represented a result favoring IAsaline. Missing standard deviations were estimated on the basis of the methods recommended in the Cochrane Handbook for Systematic Reviews of Interventions [37]. For binary outcomes, an RR less than 1 favored IAHA, while a value greater than 1 favored IAsaline. Effect size estimates were analyzed using a generic inverse variance statistical method and a random-effects analysis model with a 95\% confidence interval (CI). The number of participants who experienced a treatment-related $\mathrm{AE}$ was analyzed under a dichotomous outcome assessment using Mantel-Haenzel statistical method and a random-effects analysis model with a 95\% CI. Heterogeneity between the included trials was measured using the $I^{2}$ statistic.

Studies were stratified into two groups: (1) studies with early to moderate (early-moderate) knee OA participants (i.e., K-L grade $\leq 3$ only), and (2) studies with early to late (late) knee OA patients (i.e., the authors enrolled patients with $\mathrm{K}-\mathrm{L}$ grade 1-4 knee OA) based on the trial's demographic characteristics. If a trial had less than $5 \%$ of the patients with a baseline $\mathrm{K}-\mathrm{L}$ grade of 4 , the trial was included in the earlymoderate OA subgroup.

\section{Sensitivity Analysis}

A sensitivity analysis was conducted to determine if single-blinded studies and the nonblinded study had a significant impact on the total treatment effect of IA-HA on knee pain vs IA-saline at 13-week and 26-week follow-up periods. To accomplish this, single-blinded studies were removed from analyses to determine if they had a significant impact on treatment efficacy. Additionally, we conducted a sensitivity analysis to determine if the hand-selected study had a significant impact on the total treatment effect of IA-HA on knee pain vs IAsaline. To accomplish this, the hand-selected study was removed from analyses to determine if it had a significant impact on treatment efficacy.

\section{RESULTS}

\section{Study Characteristics and Demographics}

The sample sizes of the included trials ranged from 12 to 588 patients (Table 1). Sixteen studies $(80.0 \%)$ were double-blinded RCTs and four (20.0\%) were single-blinded. The IA-HA formulations used across these trials were Adant, Durolane, Euflexxa, Fermathron Plus, Gel-One, Hyalgan, Orthovisc, Monovisc, and Synvisc; the specific brand was not reported in two studies.

The average ages and BMIs ranged from 53 to 73 years and 25 to $33 \mathrm{~kg} / \mathrm{m}$ [2], respectively, (Table 2). Of 19 studies, 16 were included in the early-moderate OA subgroup and the other three were included in the late OA subgroup. The other study, Henderson et al. presented their results for patients with $\mathrm{K}-\mathrm{L}$ grade 2 and those with K-L grade 3-4; therefore this study was represented in both subgroups as Henderson 1994a (early-moderate OA subgroup) and Henderson 1994b (late OA subgroup) [25]. 
Table 1 Study characteristics of the included trials

\begin{tabular}{|c|c|c|c|c|c|}
\hline Study & $\begin{array}{l}\text { Sample } \\
\text { size }\end{array}$ & Countries & Study design & $\begin{array}{l}\text { IA-HA } \\
\text { formulation }\end{array}$ & $\begin{array}{l}\text { Outcome measure } \\
\text { for pain }\end{array}$ \\
\hline Altman et al. (2004) & 346 & USA, Canada, Sweden & $\begin{array}{l}\text { Double-blinded } \\
\text { RCT }\end{array}$ & Durolane & $\begin{array}{l}\text { WOMAC pain, 0-20 } \\
\text { Likert }\end{array}$ \\
\hline Altman et al. (2009) & 588 & USA & $\begin{array}{l}\text { Double-blinded } \\
\text { RCT }\end{array}$ & Euflexxa & VAS pain, $100 \mathrm{~mm}$ \\
\hline Arden et al. (2014) & 218 & Sweden, German, UK & $\begin{array}{l}\text { Double-blinded } \\
\text { RCT }\end{array}$ & Durolane & $\begin{array}{l}\text { WOMAC pain, 0-20 } \\
\text { Likert }\end{array}$ \\
\hline Brandt et al. (2001) & 226 & USA & $\begin{array}{l}\text { Double-blinded } \\
\text { RCT }\end{array}$ & Orthovisc & $\begin{array}{l}\text { WOMAC pain, 5-25 } \\
\text { Likert }\end{array}$ \\
\hline Chevalier et al. (2010) & 253 & $\begin{array}{l}\text { UK, France, Czech } \\
\text { Republic, } \\
\text { Germany, Belgium, } \\
\text { the Netherlands }\end{array}$ & $\begin{array}{l}\text { Double-blinded } \\
\text { RCT }\end{array}$ & Synvisc & $\begin{array}{l}\text { WOMAC pain, } 0-4 \\
\text { Likert }\end{array}$ \\
\hline Creamer et al. (1994) & 12 & UK & $\begin{array}{l}\text { Single-blinded } \\
\text { RCT }\end{array}$ & Hyalgan & VAS pain, $100 \mathrm{~mm}$ \\
\hline Cubukcu et al. (2005) & 40 & Turkey & $\begin{array}{l}\text { Single-blinded } \\
\text { RCT }\end{array}$ & Synvisc & $\begin{array}{l}\text { WOMAC pain, 5-25 } \\
\text { Likert }\end{array}$ \\
\hline DeCaria et al. (2012) & 30 & Canada & $\begin{array}{l}\text { Double-blinded } \\
\text { RCT }\end{array}$ & Hyalgan & $\begin{array}{l}\text { WOMAC pain, 0-20 } \\
\text { Likert }\end{array}$ \\
\hline Diracoglu et al. (2009) & 63 & Turkey & $\begin{array}{l}\text { Double-blinded } \\
\text { RCT }\end{array}$ & Synvisc & $\begin{array}{l}\text { WOMAC pain, 0-20 } \\
\text { Likert }\end{array}$ \\
\hline Hangody et al. (2017) & 368 & Europe, Canada & $\begin{array}{l}\text { Double-blinded } \\
\text { RCT }\end{array}$ & Monovisc & $\begin{array}{l}\text { WOMAC pain, } \\
100 \mathrm{~mm}\end{array}$ \\
\hline $\begin{array}{l}\text { Henderson et al. } \\
\text { (1994) }\end{array}$ & 91 & UK & $\begin{array}{l}\text { Double-blinded } \\
\text { RCT }\end{array}$ & Hyalgan & VAS pain, $100 \mathrm{~mm}$ \\
\hline Huang et al. (2011) & 200 & Taiwan & $\begin{array}{l}\text { Double-blinded } \\
\text { RCT }\end{array}$ & Hyalgan & $\begin{array}{l}\text { WOMAC pain, } \\
100 \mathrm{~mm}\end{array}$ \\
\hline Huskisson et al. (1999) & 100 & UK & $\begin{array}{l}\text { Single-blinded } \\
\text { RCT }\end{array}$ & Hyalgan & VAS pain, $100 \mathrm{~mm}$ \\
\hline $\begin{array}{l}\text { Lundsgaard et al. } \\
\qquad(2008)\end{array}$ & 308 & UK, the Netherlands & $\begin{array}{l}\text { Double-blinded } \\
\text { RCT }\end{array}$ & Hyalgan & VAS pain, $100 \mathrm{~mm}$ \\
\hline $\begin{array}{l}\text { Navarro-Sarabia et al. } \\
\text { (2011) }\end{array}$ & 306 & Spain & $\begin{array}{l}\text { Double-blinded } \\
\text { RCT }\end{array}$ & Adant & $\begin{array}{l}\text { WOMAC pain, } \\
100 \mathrm{~mm}\end{array}$ \\
\hline Neustadt et al. (2005) & 229 & USA, Canada & $\begin{array}{l}\text { Double-blinded } \\
\text { RCT }\end{array}$ & Orthovisc & $\begin{array}{l}\text { WOMAC pain, } \\
0-500\end{array}$ \\
\hline Petrella et al. (2006) & 106 & Canada & $\begin{array}{l}\text { Double-blinded } \\
\text { RCT }\end{array}$ & Suplasyn & $\begin{array}{l}\text { WOMAC pain, 5-25 } \\
\text { Likert }\end{array}$ \\
\hline
\end{tabular}


Table 1 continued

\begin{tabular}{|c|c|c|c|c|c|}
\hline$\overline{\text { Study }}$ & $\begin{array}{l}\text { Sample } \\
\text { size }\end{array}$ & Countries & Study design & $\begin{array}{l}\text { IA-HA } \\
\text { formulation }\end{array}$ & $\begin{array}{l}\text { Outcome measure } \\
\text { for pain }\end{array}$ \\
\hline Sezgin et al. (2005) & 41 & Turkey & $\begin{array}{l}\text { Single-blinded } \\
\text { RCT }\end{array}$ & Orthovisc & $\begin{array}{l}\text { WOMAC pain, 5-25 } \\
\text { Likert }\end{array}$ \\
\hline Strand et al. (2012) & 379 & Japan & $\begin{array}{l}\text { Double-blinded } \\
\text { RCT }\end{array}$ & Gel-ONE & $\begin{array}{l}\text { WOMAC pain, } \\
100 \mathrm{~mm}\end{array}$ \\
\hline $\begin{array}{l}\text { Van der Weegen et al. } \\
(2015)\end{array}$ & 196 & The Netherlands & $\begin{array}{l}\text { Double-blinded } \\
\text { RCT }\end{array}$ & $\begin{array}{l}\text { Fermathron } \\
\text { plus }\end{array}$ & VAS pain, $100 \mathrm{~mm}$ \\
\hline
\end{tabular}

\section{Pain at 4-13 Weeks (Earlier Visit)}

In the early-moderate OA subgroup (16 trials; $n=1578$ for IA-HA, $n=1341$ for saline), the mean change in pain scores from baseline within this visit window was statistically significant in favor of IA-HA [SMD $=-0.30,95 \%$ $\mathrm{CI}-0.44$ to $\left.-0.15, p<0.0001 ; I^{2}=68 \%\right]$ (Fig. 2). In the late OA subgroup (4 trials; $n=288$ for IAHA, $n=278$ for saline), there was no significant effect between IA-HA and saline $[\mathrm{SMD}=0.28,95 \% \mathrm{CI}-0.12$ to $0.68, p=0.17$; $\left.I^{2}=72 \%\right]$.

The test for subgroup differences (earlymoderate $\mathrm{OA}$ versus late $\mathrm{OA}$ ) was statistically significant $(p=0.008)$. The funnel plot revealed no evidence of publication bias (Fig. 3).

\section{Pain at 22-27 Weeks (Later Visit)}

In the early-moderate OA subgroup (9 trials; $n=1058$ for IA-HA, $n=981$ for saline), the mean change in pain scores from baseline within this visit window was statistically significant in favor of IA-HA [SMD $=-0.27,95 \%$ CI -0.39 to $\left.-0.16, p<0.00001 ; I^{2}=38 \%\right]$ (Fig. 4). In the late OA subgroup ( 2 trials; $n=254$ for IAHA, $n=254$ for saline), there was no significant effect between IA-HA and saline $[\mathrm{SMD}=0.03,95 \% \mathrm{CI}-0.14$ to $0.21, p=0.72$; $\left.I^{2}=3 \%\right]$.

The test for subgroup differences (earlymoderate $\mathrm{OA}$ versus late $\mathrm{OA}$ ) was statistically significant $(p=0.005)$. The funnel plot revealed no evidence of publication bias (Fig. 5).

\section{Treatment-Related AEs}

In the early-moderate OA subgroup ( 9 trials; $n=1304$ for IA-HA, $n=1104$ for saline), there was no significant difference in the risk of treatment-related AEs between IA-HA and saline injections $[\mathrm{RR}=1.03,95 \%$ CI $0.89-1.20$, $p=0.68 ; I^{2}=36 \%$ ] (Fig. 6). In the late OA subgroup ( 3 trials; $n=230$ for IAHA, $n=232$ for saline), IA-HA was associated with a significantly greater risk of treatment-related AEs relative to saline $[R R=1.76,95 \%$ CI $1.16-2.67$, $\left.p=0.008 ; I^{2}=0 \%\right]$.

The test for subgroup differences (earlymoderate $\mathrm{OA}$ versus late $\mathrm{OA})$ was statistically significant $(p=0.02)$.

\section{Sensitivity Analysis}

Three single-blinded studies and one non-blinded were removed in the sensitivity analysis $[23,24,29,34]$. The pooled effect size remained statistically significant with little change in total effect size at 13-week (SMD $=-0.16[-0.32$, $-0.01], P=0.004)$ and 26-week (SMD $=-0.19$ $[-0.30,-0.07], P=0.001)$ follow-up periods.

At 13-week and 26-week follow-up periods, the pooled effect size remained statistically significant with little change in total effect size when the hand-selected study was removed from the analysis. Similar results were observed when single-blinded studies were removed from analyses; total treatment efficacy and subgroup differences remained statistically significant at 13 weeks and 26 weeks. 
Table 2 Demographic characteristics of the included trials

\begin{tabular}{|c|c|c|c|c|c|c|c|c|c|c|}
\hline \multirow[t]{2}{*}{ Study } & \multicolumn{5}{|c|}{ IA-HA arm } & \multicolumn{5}{|c|}{ Control arm } \\
\hline & $n$ & $\begin{array}{l}\text { Mean } \\
\text { age }\end{array}$ & $\begin{array}{l}\text { Mean } \\
\text { BMI }\end{array}$ & $\begin{array}{l}\% \\
\text { male }\end{array}$ & $\begin{array}{l}\text { K-L grade, } \\
(\%)\end{array}$ & $n$ & $\begin{array}{l}\text { Mean } \\
\text { age }\end{array}$ & $\begin{array}{l}\text { Mean } \\
\text { BMI }\end{array}$ & $\begin{array}{l}\% \\
\text { male }\end{array}$ & $\begin{array}{l}\text { K-L grade, } \\
n(\%)\end{array}$ \\
\hline \multirow[t]{2}{*}{ Altman (2004) } & 172 & 62.9 & M: 29.5 & 54.1 & $1-3: 76.7$ & 174 & 63.3 & M: 29.0 & 36.2 & $1-3: 74.1$ \\
\hline & & & F: 31.3 & & 4: 23.3 & & & F: 29.8 & & 4: 25.9 \\
\hline \multirow[t]{2}{*}{ Altman (2009) } & 291 & 62.5 & 32.4 & 37.0 & $1-3: 100.0$ & 295 & 60.8 & 33.0 & 37.0 & $1-3: 100.0$ \\
\hline & & & & & 4: 0.0 & & & & & 4: 0.0 \\
\hline \multirow[t]{2}{*}{ Arden (2014) } & 108 & 64.5 & M: 28.2 & 45.0 & 1-3: 100.0 & 110 & 60.9 & M: 28.1 & 54.0 & 1-3: 100.0 \\
\hline & & & F: 26.4 & & 4: 0.0 & & & F: 26.9 & & 4: 0.0 \\
\hline \multirow[t]{2}{*}{ Brandt (2001) } & 114 & 65.0 & 32.0 & 37.0 & $1-3: 100.0$ & 112 & 67.0 & 30.1 & 37.0 & $1-3: 100.0$ \\
\hline & & & & & 4: 0.0 & & & & & 4: 0.0 \\
\hline \multirow[t]{2}{*}{ Chevalier (2010) } & 124 & 63.6 & 29.1 & 25.8 & $1-3: 100.0$ & 129 & 62.5 & 29.8 & 31.8 & $1-3: 99.2$ \\
\hline & & & & & 4: 0.0 & & & & & 4: 0.8 \\
\hline \multirow[t]{2}{*}{ Creamer (1994) } & 12 & NR & NR & 0.0 & $1-3: 66.7$ & 12 & NR & NR & 0.0 & $1-3: 66.7$ \\
\hline & & & & & 4: 33.3 & & & & & 4: 33.3 \\
\hline \multirow[t]{2}{*}{ Cubukcu (2005) } & 30 & 52.6 & NR & 30.0 & $1-3: 100.0$ & 10 & 57.6 & NR & 0.0 & $1-3: 100.0$ \\
\hline & & & & & 4: 0.0 & & & & & 4: 0.0 \\
\hline \multirow[t]{2}{*}{ DeCaria (2012) } & 15 & 71.9 & 30.5 & 53.0 & $1-3: 100.0$ & 15 & 72.9 & 29.4 & 53.0 & $1-3: 100.0$ \\
\hline & & & & & 4: 0.0 & & & & & 4: 0.0 \\
\hline \multirow[t]{2}{*}{ Diracoglu (2009) } & 42 & 59.4 & 31.1 & 10.0 & 1-3: 100.0 & 21 & 56.2 & 31.3 & 0.0 & 1-3: 100.0 \\
\hline & & & & & 4: 0.0 & & & & & 4: 0.0 \\
\hline \multirow[t]{2}{*}{ Hangody (2017) } & 150 & 59.2 & 28.4 & 34.0 & 1-3: 99.3 & 69 & 58.0 & 29.1 & 26.1 & 1-3: 100.0 \\
\hline & & & & & 4: 0.7 & & & & & 4: 0.0 \\
\hline \multirow[t]{2}{*}{ Henderson (1994) } & 40 & NR & NR & NR & $1-3: 82.2$ & 44 & NR & NR & NR & $1-3: 71.7$ \\
\hline & & & & & 4: 17.8 & & & & & 4: 28.3 \\
\hline \multirow[t]{2}{*}{ Huang (2011) } & 100 & 65.9 & 25.7 & 26.0 & $1-3: 100.0$ & 100 & 64.2 & 25.4 & 22.0 & $1-3: 100.0$ \\
\hline & & & & & 4: 0.0 & & & & & 4: 0.0 \\
\hline \multirow[t]{2}{*}{ Huskisson (1999) } & 50 & 65.8 & NR & 24.0 & $1-3: 100.0$ & 50 & 64.8 & NR & 42.0 & $1-3: 100.0$ \\
\hline & & & & & 4: 0.0 & & & & & 4: 0.0 \\
\hline \multirow[t]{2}{*}{ Lundsgaard (2008) } & 82 & 68.8 & 29.6 & 42.9 & $1-3: 63.1$ & 80 & 69.6 & 29.3 & 47.6 & $1-3: 61.2$ \\
\hline & & & & & 4: 36.9 & & & & & 4: 38.8 \\
\hline \multirow{2}{*}{$\begin{array}{l}\text { Navarro-Sarabia } \\
\text { (2011) }\end{array}$} & 153 & 63.0 & 28.4 & 16.3 & 1-3: 100.0 & 153 & 63.9 & 28.7 & 16.3 & 1-3: 100.0 \\
\hline & & & & & 4: 0.0 & & & & & 4: 0.0 \\
\hline
\end{tabular}


Table 2 continued

\begin{tabular}{|c|c|c|c|c|c|c|c|c|c|c|}
\hline \multirow[t]{2}{*}{ Study } & \multicolumn{5}{|c|}{ IA-HA arm } & \multicolumn{5}{|c|}{ Control arm } \\
\hline & $n$ & $\begin{array}{l}\text { Mean } \\
\text { age }\end{array}$ & $\begin{array}{l}\text { Mean } \\
\text { BMI }\end{array}$ & $\begin{array}{l}\% \\
\text { male }\end{array}$ & $\begin{array}{l}\text { K-L grade, } \\
(\%)\end{array}$ & $n$ & $\begin{array}{l}\text { Mean } \\
\text { age }\end{array}$ & $\begin{array}{l}\text { Mean } \\
\text { BMI }\end{array}$ & $\begin{array}{l}\text { \% } \\
\text { male }\end{array}$ & $\begin{array}{l}\text { K-L grade, } \\
n(\%)\end{array}$ \\
\hline Neustadt (2005) & 115 & 58.4 & 28.9 & 54.8 & $\begin{array}{l}1-3: 100.0 \\
4: 0.0\end{array}$ & 114 & 59.1 & 29.4 & 50.0 & $\begin{array}{l}1-3: 100.0 \\
4: 0.0\end{array}$ \\
\hline Petrella (2006) & 53 & 63.9 & NR & 56.9 & $\begin{array}{l}1-3: 100.0 \\
4: 0.0\end{array}$ & 53 & 62.4 & NR & 53.7 & $\begin{array}{l}1-3: 100.0 \\
4: 0.0\end{array}$ \\
\hline Sezgin (2005) & 22 & 59.9 & 30.2 & 18.2 & $\begin{array}{l}1-3: 100.0 \\
4: 0.0\end{array}$ & 19 & 59.4 & 29.3 & 31.6 & $\begin{array}{l}1-3: 100.0 \\
4: 0.0\end{array}$ \\
\hline Strand (2012) & 247 & 60.9 & 28.3 & 40.5 & $\begin{array}{l}1-3: 100.0 \\
4: 0.0\end{array}$ & 128 & 60.3 & 28.7 & 39.8 & $\begin{array}{l}1-3: 100.0 \\
4: 0.0\end{array}$ \\
\hline $\begin{array}{l}\text { Van der Weegen } \\
\quad(2015)\end{array}$ & 99 & 58.7 & 28.6 & 49 & $\begin{array}{l}1-3: 100.0 \\
4: 0.0\end{array}$ & 97 & 60.1 & 29.3 & 50 & $\begin{array}{l}1-3: 100.0 \\
4: 0.0\end{array}$ \\
\hline
\end{tabular}

$N R$ not reported

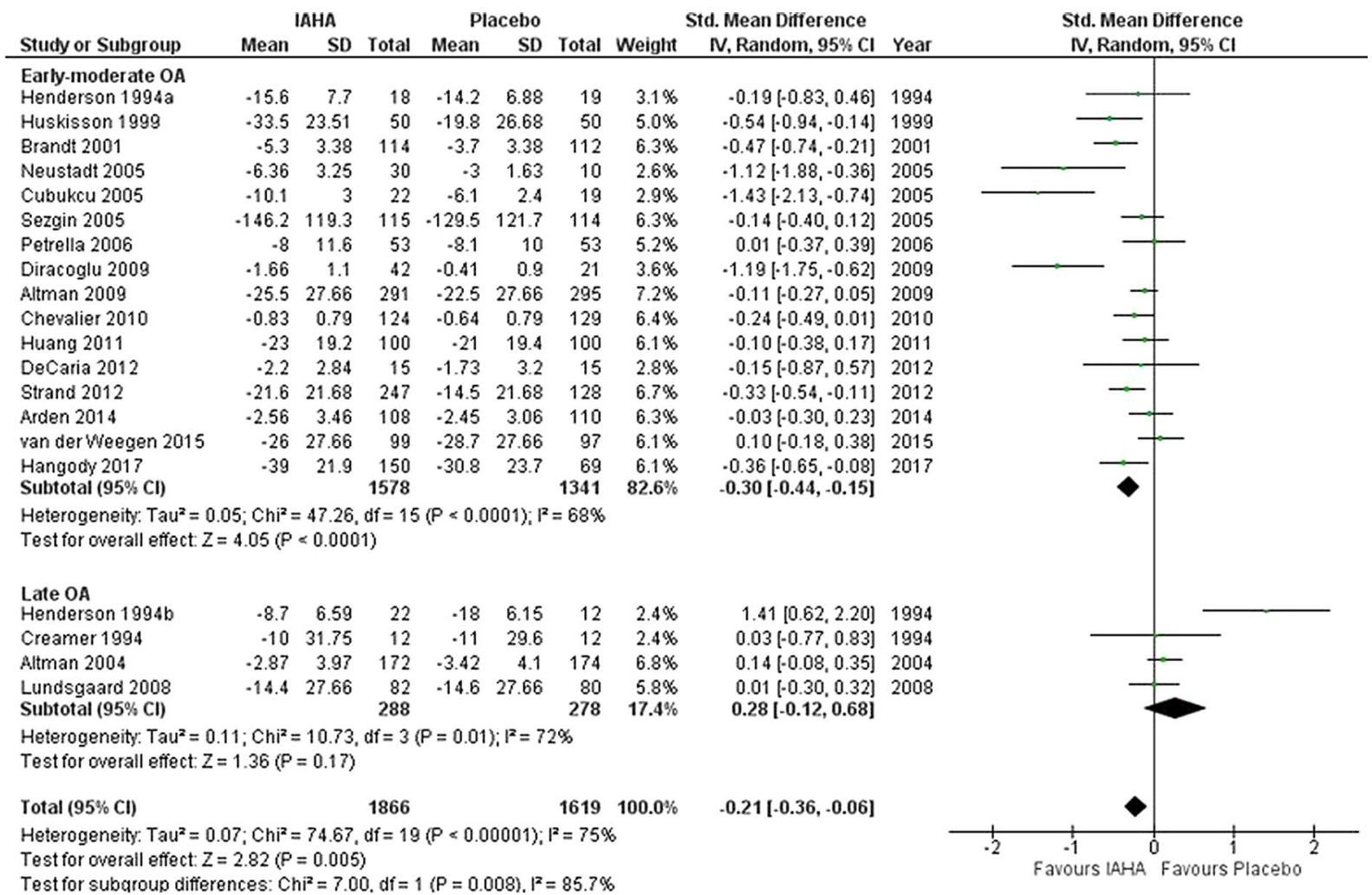

Fig. 2 Pain at 4-13 weeks 


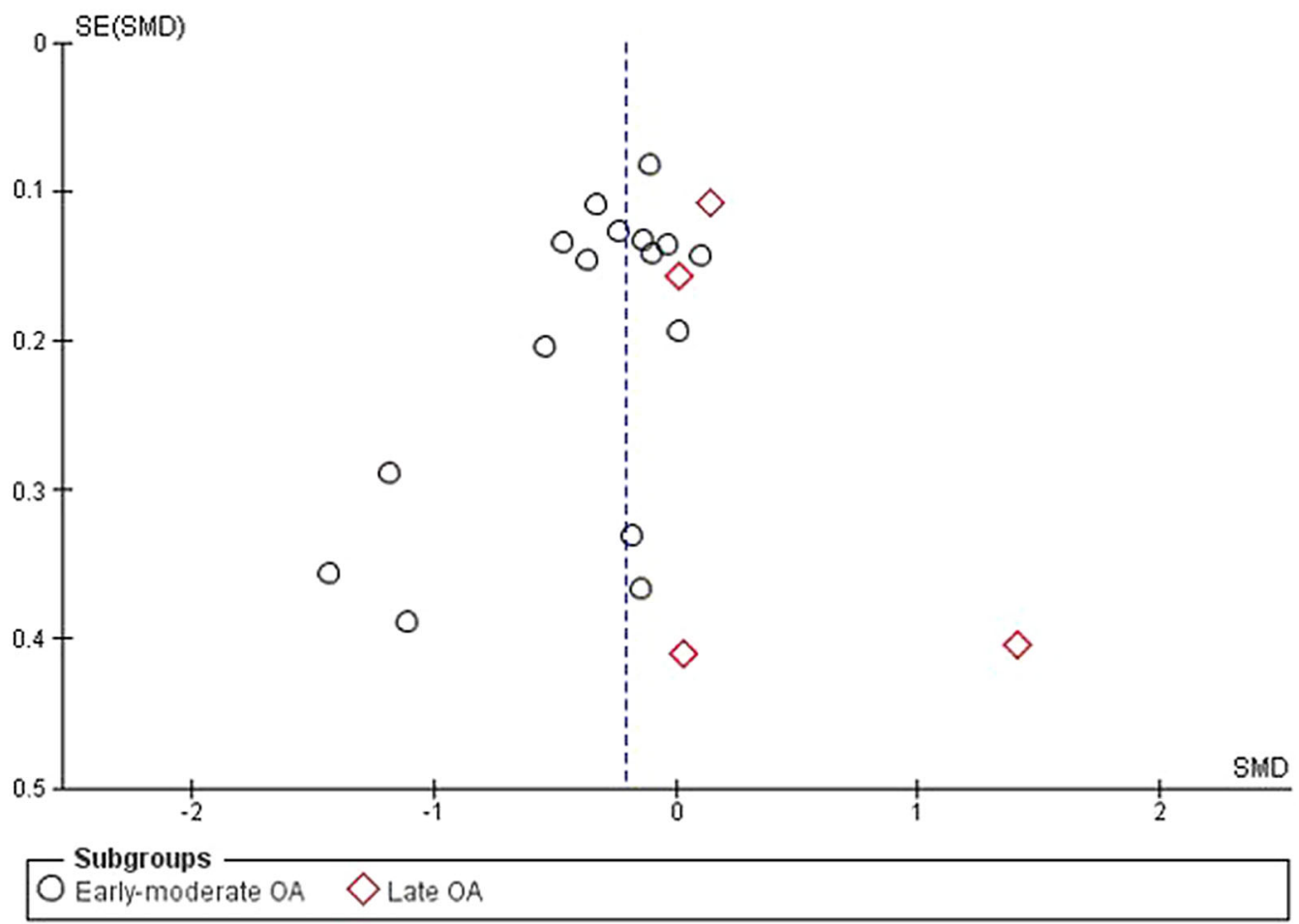

Fig. 3 Funnel plot for pain at 4-13 weeks

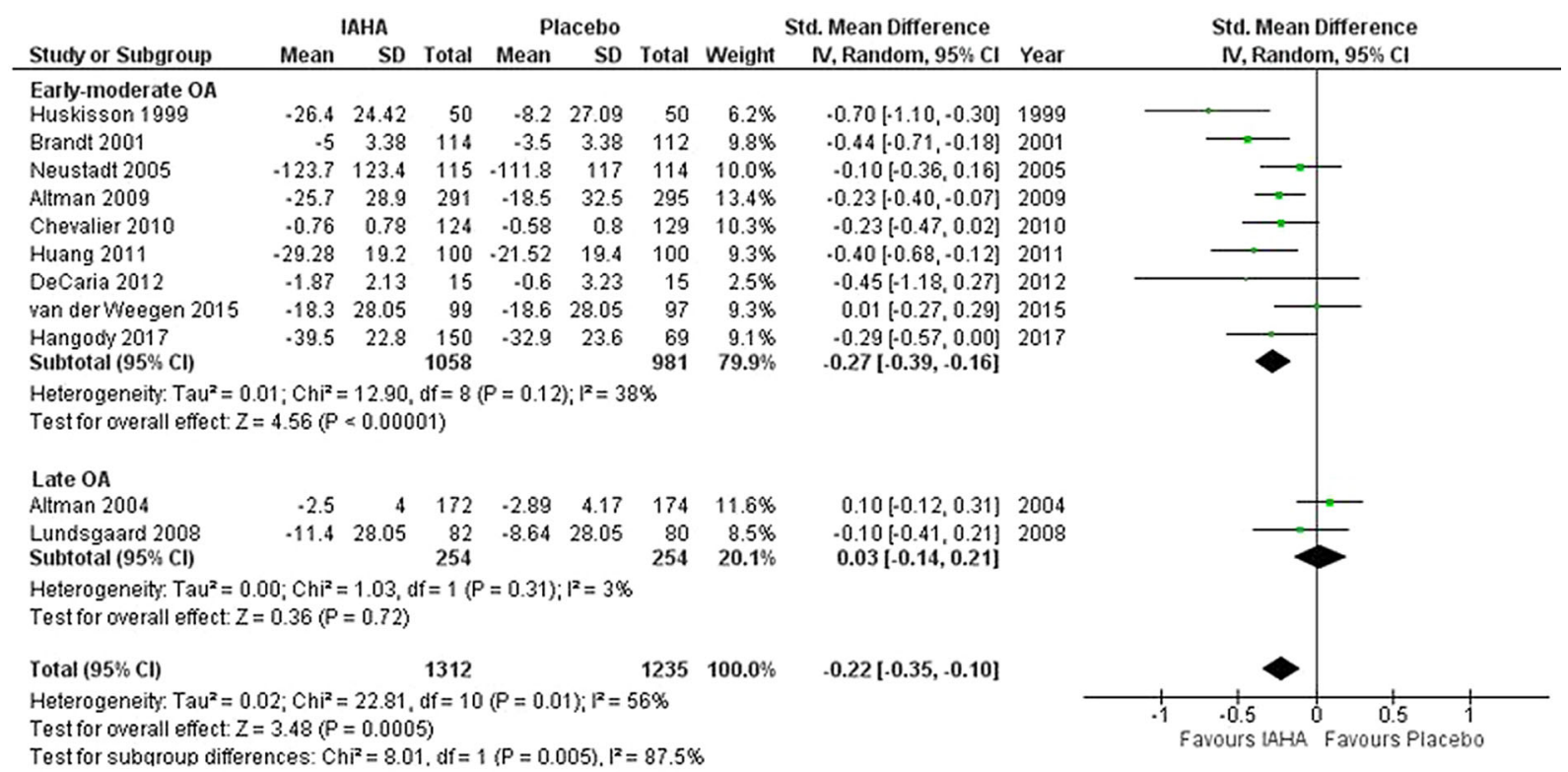

Fig. 4 Pain at 22-27 weeks

\section{DISCUSSION}

The results of this meta-analysis suggest that IAHA therapy is most efficacious in reducing pain

in patients with early-moderate knee OA, but not in the late OA subgroup. This analysis also revealed that significant pain relief with HA injections can occur within 4-13 weeks (earlier 


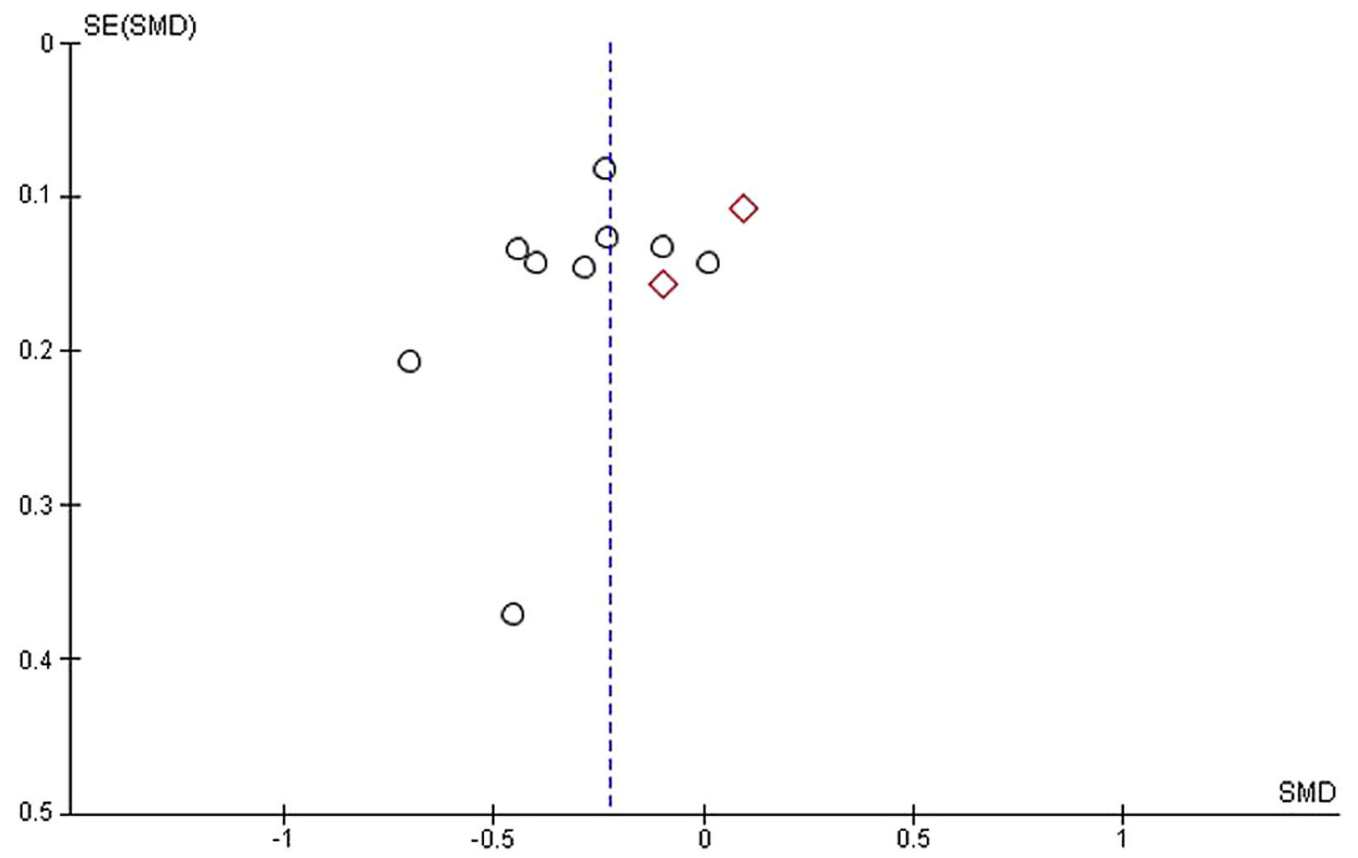

Subgroups

$O$ Early-moderate $O A \otimes$ Late $O A$

Fig. 5 Funnel plot for pain at 22-27 weeks

IAHA Placebo Risk Ratio

\begin{tabular}{lrrrrr} 
Study or Subgroup & Events & Total & Events & Total & Weight \\
\hline Early-moderate OA & & & & & \\
Huskisson 1999 & 2 & 50 & 0 & 50 & $0.3 \%$ \\
Brandt 2001 & 76 & 114 & 74 & 112 & $20.3 \%$ \\
Neustadt 2005 & 11 & 128 & 4 & 123 & $2.2 \%$ \\
Altman 2009 & 157 & 293 & 169 & 295 & $22.3 \%$ \\
Chevalier 2010 & 70 & 123 & 79 & 130 & $19.2 \%$ \\
Navarro-Sarabia 2011 & 15 & 153 & 14 & 153 & $5.1 \%$ \\
Strand 2012 & 67 & 249 & 33 & 128 & $12.5 \%$ \\
Arden 2014 & 17 & 44 & 6 & 44 & $3.8 \%$ \\
Hangody 2017 & 3 & 150 & 0 & 69 & $0.3 \%$ \\
Subtotal (95\% Cl) & & 1304 & & 1104 & $86.1 \%$ \\
Total events & 418 & & 379 & & \\
Heterogeneity Tau & $0.01 ;$ &
\end{tabular}

Heterogeneity: $\operatorname{Tau}^{2}=0.01 ; \mathrm{Chi}^{2}=12.41, \mathrm{df}=8(P=0.13) ; \mathrm{I}^{2}=36 \%$

Test for overall effect: $Z=0.41(P=0.68)$

\section{Late $O A$}

Creamer 1994

Henderson 1994

Altman 2004

Subtotal $(95 \% \mathrm{CI})$

Total events

Heterogeneity: $\mathrm{Tau}^{2}=0.00 ; \mathrm{Chi}^{2}=0.70, \mathrm{di}=2(\mathrm{P}=0.70) ; \mathrm{F}^{2}=0 \%$

Test for overall effect: $Z=2.67(P=0.008)$

Total $(95 \% \mathrm{Cl})$

Total events

1534 466

$1336100.0 \%$ 407

Heterogeneity: $\mathrm{Tau}^{2}=0.03 ; \mathrm{Chi}^{2}=20.90, \mathrm{df}=11(P=0.03) ; \mathrm{I}^{2}=47 \%$

Test for overall effect $Z=1.49(P=0.14)$

Test for subaroup differences: $\mathrm{Ch}^{2}=5.63, \mathrm{df}=1(\mathrm{P}=0.02), \mathrm{I}^{2}=82.2 \%$

Fig. 6 Treatment-related AE $1.01[0.84,1.21] 2001$

$2.64[0.86,8.08] 2005$

$0.94[0.81,1.08] 2009$

$0.94[0.76,1.15] 2010$

$1.07[0.54,2.14] 2011$

$1.04[0.73,1.49] 2012$

$2.83[1.23,6.51] 2014$

$3.25[0.17 .61 .97] 2017$

$1.03[0.89,1.20]$

$1.67[0.51,5.46] \quad 1994$ $2.15[1.14,4.03] 1994$

$1.48[0.79,2.75] 2004$

$1.76[1.16,2.67]$

$1.14[0.96,1.36]$

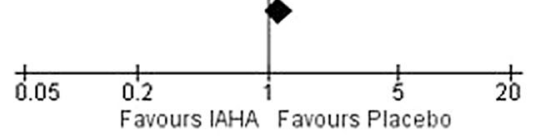


visit) post-injection and remain beneficial up to approximately 6 months (later visit); the effect estimates were similar between these two time points (SMD $=-0.30,95 \% \mathrm{CI}-0.44$ to -0.15 at the earlier visit and SMD $=-0.27,95 \% \mathrm{CI}$ -0.39 to -0.16 at the later visit). This suggests that the effect seen with HA plateaus and is maintained up to 6 months post-injection.

In 2015, Strand et al. published a metaanalysis evaluating the effects of IA-HA within subcategories of disease severity [16]. Similar to the current study, Strand et al. found that the effect size for pain between IA-HA and saline injections in the early-moderate OA subgroup $(\mathrm{K}-\mathrm{L}$ grade $\leq 3)$ was statistically significant $(\mathrm{SMD}=-0.35,95 \% \mathrm{CI}-0.57$ to -0.14$)$, but this outcome was not significant in the subgroup that also included $\mathrm{K}-\mathrm{L}$ grade 4 patients $(\mathrm{SMD}=-0.11,95 \% \mathrm{CI}-0.46$ to 0.24$)$. Other SMDs reported in previous meta-analyses, without considering OA grade, have ranged from -0.19 to $-0.43[10,16,38-42]$. The estimates that were calculated in the current analysis for the early-moderate OA subgroup $(-0.30$ at $4-13$ weeks and -0.27 at $22-$ 27 weeks) were within this range, but those for the late OA subgroup (0.28 at 4-13 weeks and 0.03 at 22-27 weeks) were not. Such an observation may be explained by the fact that most IA-HA studies have only included patients with early to moderate knee OA [4]. The current study provides further quantitative evidence that including patients with $\mathrm{K}-\mathrm{L}$ grade 4 knee OA dilutes the benefit of HA injections when averaging treatment effects within a sample. These results also demonstrated the potential for HA to provide greater benefit to patients if it is provided earlier in the course of their disease, as opposed to being provided as a later treatment option once disease severity has already progressed to late stages. These results also demonstrate that the late OA subgroup experienced significantly more treatment-related AEs, suggesting that patients with KL grade 4 may be more susceptible to adverse events. These results may have been influenced by the inclusion of avian-derived HA and non-animal stabilized HA formulations, as well as the use of low molecular HA. Further insight into the product differences of IA-HA in KL grade 4 participants is required.

Several knee OA guidelines are available to practicing physicians; however, there is clearly inconsistency in the recommendations for IAHA injections [4, 5, 43]. What many of these guidelines fail to address is how the efficacy of IA-HA may vary by select patient characteristics. The results of this systematic review suggest that future clinical practice guidelines base their treatment recommendations on the individual patient's disease state and focus on the potential for greater benefit when IA-HA is provided in earlier stage knee OA.

A limitation of the current study is the heterogeneity within subgroups, suggesting that there may be other factors contributing to the difference in results between the subgroups. It has been previously established that IA-HA trials are variable in terms of patient eligibility criteria, HA molecular characteristics, injection schedules, and outcomes assessment [3], which must also be further investigated. Additionally, there is a small proportion of included patients who were in advanced stages of their knee OA. Although this proportion is small, the results of this study still provide valuable information regarding the potential effects seen in these late stage patients. Studies included in this analysis varied in terms of blinding, HA product (with different injection regimens and molecular weights), and used either the VAS for pain or WOMAC pain subscale, though there is even variability in the subscale for WOMAC pain (e.g., the $0-100 \mathrm{~mm}, 0-20$ or 5-25 Likert, or $0-500$ scale). The $\mathrm{K}-\mathrm{L}$ grade is the most widely used OA severity scale; however, other measures exist, with their own definitions and scoring systems [1], and studies that reported relevant outcomes were excluded from this analysis for this reason. There is also controversy as to whether or not radiographic criteria always correlate with clinical symptoms [1]. It cannot be said, definitely, that patients with more severe OA based on radiographs are also more symptomatic at baseline. Non-English trials were excluded, which may limit the generalizability of these findings and confidence in the effect estimates. The included studies were predominantly representative of sites in North 
America and Europe, though two trials were conducted in Asia [26, 33] and demonstrated comparable treatment effects for HA. Lastly, the number of studied including participants with KL grade 4 knee OA was limited, suggesting that this cohort may not be actively recruited in IAHA clinical trials.

A strength of this analysis was that only data from randomized, saline-controlled trials were included, ensuring that pooled effects estimates were from evidence of high-quality studies with a common comparator. There was also a large number patients in the analysis $(n=3485$ in the assessment of pain at the earlier visit window), though a substantial proportion of this sample represented patients in the early-moderate OA subgroup. The outcome scales used to assess pain (i.e., the VAS and WOMAC) are validated measures and commonly evaluated in the knee OA literature. The funnel plots also revealed that publication bias is unlikely.

\section{CONCLUSIONS}

Treatment with IA-HA provides statistically significant pain relief compared to saline injections for patients with early-moderate knee OA, with no increase in the risk of treatment-related adverse effects, up to 6 months post-injection. IA-HA demonstrated no benefit over controls in the late OA subgroup and was associated with significantly greater treatment-related AEs. In this regard, some of the prior studies that demonstrated no significant benefit with HA injections may have confounded their results with the inclusion of a considerable proportion of patients with end-stage knee disease. Future investigations on the topic should take caution in completely rejecting the potential benefit of IA-HA when it may indeed be efficacious for a subset of the knee OA population.

\section{ACKNOWLEDGEMENTS}

Funding. This study was funded by Ferring Pharmaceuticals, including funding of medical writing, article processing charges, and Open
Access fees. All authors had full access to all of the data in this study and take complete responsibility for the integrity of the data and accuracy of the data analysis.

Medical Writing and Editorial Assistance. The authors would like to thank Mark Phillips of Global Research Solutions Inc. for their medical writing assistance and editorial support.

Authorship. All named authors meet the International Committee of Medical Journal Editors (ICMJE) criteria for authorship for this article, take responsibility for the integrity of the work as a whole, and have given their approval for this version to be published.

Disclosures. Mathew Nicholls is a consultant for Ferring Pharmaceuticals, Inc. Peter Shaw is a paid employee of Ferring Pharmaceuticals. Faizan Niazi is a paid employee of Ferring Pharmaceuticals. Mohit Bhandari has received fees for speaking for/organizing an educational program, as well as funds for research from Ferring Pharmaceuticals, and has an affiliation with organizations not related to this particular study, including Stryker, DJO, Acumed, Sanofi, and Pendopharm. Asheesh Bedi has nothing to disclose.

Compliance with Ethics Guidelines. This article is based on previously conducted studies and does not contain any studies with human participants or animals performed by any of the authors.

Data Availability. The datasets generated and/or analyzed during the current study are available from the corresponding author on reasonable request.

Open Access. This article is distributed under the terms of the Creative Commons Attribution-NonCommercial 4.0 International License (http://creativecommons.org/licenses/ by-nc/4.0/), which permits any noncommercial use, distribution, and reproduction in any medium, provided you give appropriate credit to the original author(s) and the source, provide 
a link to the Creative Commons license, and indicate if changes were made.

\section{REFERENCES}

1. Litwic A, Edwards M, Dennison E, Cooper C. Epidemiology and burden of osteoarthritis. Br Med Bull. 2013;105:185-99.

2. Zhang Y, Jordan JM. Epidemiology of osteoarthritis. Clin Geriatr Med. 2010;26(3):355-69.

3. Ayhan E, Kesmezacar H, Akgun I. Intraarticular injections (corticosteroid, hyaluronic acid, platelet rich plasma) for the knee osteoarthritis. World J Orthop. 2014;5(3):351-61.

4. Bhadra AK, Altman R, Dasa V, et al. Appropriate use criteria for hyaluronic acid in the treatment of knee osteoarthritis in the United States. Cartilage. 2017;8(3):234-54.

5. Altman RD, Schemitsch E, Bedi A. Assessment of clinical practice guideline methodology for the treatment of knee osteoarthritis with intra-articular hyaluronic acid. Semin Arthritis Rheum. 2015;45(2): 132-9.

6. Bruyere $\mathrm{O}$, Cooper C, Pelletier JP, et al. A consensus statement on the European Society for Clinical and Economic Aspects of Osteoporosis and Osteoarthritis (ESCEO) algorithm for the management of knee osteoarthritis-From evidence-based medicine to the real-life setting. Semin Arthritis Rheum. 2016;45(4):S3-11.

7. Jordan KM, Arden NK, Doherty M, et al. EULAR recommendations 2003: an evidence based approach to the management of knee osteoarthritis: report of a Task Force of the Standing Committee for International Clinical Studies Including Therapeutic Trials (ESCISIT). Ann Rheum Dis. 2003;62(12):1145-55.

8. Wen DY. Intra-articular hyaluronic acid injections for knee osteoarthritis. Am Fam Physician. 2000;62(3):565-70.

9. Evanich JD, Evanich CJ, Wright MB, Rydlewicz JA. Efficacy of intraarticular hyaluronic acid injections in knee osteoarthritis. Clin Orthop Relat Res. 2001;390:173-81.

10. Rutjes AW, Juni P, da Costa BR, Trelle S, Nuesch E, Reichenbach S. Viscosupplementation for osteoarthritis of the knee: a systematic review and meta-analysis. Ann Intern Med. 2012;157(3):180-91.
11. Altman RD, Bedi A, Karlsson J, Sancheti P, Schemitsch E. Product differences in intra-articular hyaluronic acids for osteoarthritis of the knee. Am J Sports Med. 2016;44(8):2158-65.

12. Kellgren JH, Lawrence JS. Radiological assessment of osteo-arthrosis. Ann Rheum Dis. 1957;16(4):494-502.

13. Gormeli G, Gormeli CA, Ataoglu B, Colak C, Aslanturk O, Ertem K. Multiple PRP injections are more effective than single injections and hyaluronic acid in knees with early osteoarthritis: a randomized, double-blind, placebo-controlled trial. Knee Surg Sports Traumatol Arthrosc. 2017;25(3):958-65.

14. Altman RD, Rosen JE, Bloch DA, Hatoum HT, Korner P. A double-blind, randomized, saline-controlled study of the efficacy and safety of EUFLEXXA for treatment of painful osteoarthritis of the knee, with an open-label safety extension (the FLEXX trial). Semin Arthritis Rheum. 2009;39(1):1-9.

15. Eymard F, Chevalier X, Conrozier T. Obesity and radiological severity are associated with viscosupplementation failure in patients with knee osteoarthritis. J Orthop Res. 2017;35(10):2269-74.

16. Strand V, McIntyre LF, Beach WR, Miller LE, Block JE. Safety and efficacy of US-approved viscosupplements for knee osteoarthritis: a systematic review and meta-analysis of randomized, saline-controlled trials. J Pain Res. 2015;8:217-28.

17. Altman RD, Akermark C, Beaulieu AD, Schnitzer T. Efficacy and safety of a single intra-articular injection of non-animal stabilized hyaluronic acid (NASHA) in patients with osteoarthritis of the knee. Osteoarthr Cartil. 2004;12(8):642-9.

18. Arden NK, Akermark C, Andersson M, Todman MG, Altman RD. A randomized saline-controlled trial of NASHA hyaluronic acid for knee osteoarthritis. Curr Med Res Opin. 2014;30(2):279-86.

19. Brandt KD, Block JA, Michalski JP, Moreland LW, Caldwell JR, Lavin PT. Efficacy and safety of intraarticular sodium hyaluronate in knee osteoarthritis. ORTHOVISC Study Group. Clin Orthop Relat Res. 2001;385:130-43.

20. Chevalier X, Jerosch J, Goupille P, et al. Single, intra-articular treatment with $6 \mathrm{ml}$ hylan G-F 20 in patients with symptomatic primary osteoarthritis of the knee: a randomised, multicentre, double-blind, placebo controlled trial. Ann Rheum Dis. 2010;69(1):113-9.

21. Creamer P, Sharif M, George E, et al. Intra-articular hyaluronic acid in osteoarthritis of the knee: an 
investigation into mechanisms of action. Osteoarthr Cartil. 1994;2(2):133-40.

22. Cubukcu D, Ardic F, Karabulut N, Topuz O. Hylan G-F 20 efficacy on articular cartilage quality in patients with knee osteoarthritis: clinical and MRI assessment. Clin Rheumatol. 2005;24(4):336-41.

23. DeCaria JE, Montero-Odasso M, Wolfe D, Chesworth BM, Petrella RJ. The effect of intra-articular hyaluronic acid treatment on gait velocity in older knee osteoarthritis patients: a randomized, controlled study. Arch Gerontol Geriatr. 2012;55(2):310-5.

24. Diracoglu D, Vural M, Baskent A, Dikici F, Aksoy C. The effect of viscosupplementation on neuromuscular control of the knee in patients with osteoarthritis. J Back Musculoskelet Rehabil. 2009;22(1):1-9.

25. Henderson EB, Smith EC, Pegley F, Blake DR. Intraarticular injections of $750 \mathrm{kD}$ hyaluronan in the treatment of osteoarthritis: a randomised single centre double-blind placebo-controlled trial of 91 patients demonstrating lack of efficacy. Ann Rheum Dis. 1994;53(8):529-34.

26. Huang TL, Chang CC, Lee $\mathrm{CH}$, Chen SC, Lai CH, Tsai CL. Intra-articular injections of sodium hyaluronate (Hyalgan $\left.{ }^{\circledR}\right)$ in osteoarthritis of the knee. a randomized, controlled, double-blind, multicenter trial in the Asian population. BMC Musculoskelet Disord. 2011;12:221.

27. Huskisson EC, Donnelly S. Hyaluronic acid in the treatment of osteoarthritis of the knee. Rheumatology (Oxford). 1999;38(7):602-7.

28. Lundsgaard C, Dufour N, Fallentin E, Winkel P, Gluud C. Intra-articular sodium hyaluronate $2 \mathrm{~mL}$ versus physiological saline $20 \mathrm{~mL}$ versus physiological saline $2 \mathrm{~mL}$ for painful knee osteoarthritis: a randomized clinical trial. Scand J Rheumatol. 2008;37(2):142-50.

29. Navarro-Sarabia F, Coronel P, Collantes E, et al. A 40-month multicentre, randomised placebo-controlled study to assess the efficacy and carry-over effect of repeated intra-articular injections of hyaluronic acid in knee osteoarthritis: the AMELIA project. Ann Rheum Dis. 2011;70(11):1957-62.

30. Neustadt D, Caldwell J, Bell M, Wade J, Gimbel J. Clinical effects of intraarticular injection of high molecular weight hyaluronan (Orthovisc) in osteoarthritis of the knee: a randomized, controlled, multicenter trial. J Rheumatol. 2005;32(10):1928-36.

31. Petrella RJ, Petrella M. A prospective, randomized, double-blind, placebo controlled study to evaluate the efficacy of intraarticular hyaluronic acid for osteoarthritis of the knee. J Rheumatol. 2006;33(5):951-6.

32. Sezgin M, Demirel AC, Karaca C, et al. Does hyaluronan affect inflammatory cytokines in knee osteoarthritis? Rheumatol Int. 2005;25(4):264-9.

33. Strand V, Baraf HS, Lavin PT, Lim S, Hosokawa H. A multicenter, randomized controlled trial comparing a single intra-articular injection of Gel-200, a new cross-linked formulation of hyaluronic acid, to phosphate buffered saline for treatment of osteoarthritis of the knee. Osteoarthr Cartil. 2012;20(5):350-6.

34. van der Weegen W, Wullems JA, Bos E, Noten H, van Drumpt RA. No difference between intra-articular injection of hyaluronic acid and placebo for mild to moderate knee osteoarthritis: a randomized, controlled, double-blind trial. J Arthroplasty. 2015;30(5):754-7.

35. Hangody L, Szody R, Lukasik P, et al. Intraarticular injection of a cross-linked sodium hyaluronate combined with triamcinolone hexacetonide (Cingal) to provide symptomatic relief of osteoarthritis of the knee: a randomized, double-blind, placebo-controlled multicenter clinical trial. Cartilage. 2018;9(3):276-83. https://doi.org/10.1177/1947603517703732

36. Review Manager (RevMan) [computer program]. Version 5.3. Copenhagen: The Nordic Cochrane Centre2014.

37. Cochrane handbook for systematic reviews of interventions version 5.1.0. The Cochrane Collaboration; 2011.

38. Bannuru RR, Natov NS, Dasi UR, Schmid CH, McAlindon TE. Therapeutic trajectory following intra-articular hyaluronic acid injection in knee osteoarthritis-meta-analysis. Osteoarthr Cartil. 2011;19(6):611-9.

39. Bannuru RR, Schmid CH, Kent DM, Vaysbrot EE, Wong JB, McAlindon TE. Comparative effectiveness of pharmacologic interventions for knee osteoarthritis: a systematic review and network meta-analysis. Ann Intern Med. 2015;162(1):46-54.

40. Lo GH, LaValley M, McAlindon T, Felson DT. Intraarticular hyaluronic acid in treatment of knee osteoarthritis: a meta-analysis. JAMA. 2003;290(23):3115-21.

41. Richette P, Chevalier X, Ea HK, et al. Hyaluronan for knee osteoarthritis: an updated meta-analysis of trials with low risk of bias. RMD Open. 2015;1(1):e000071. 
42. Trojian TH, Concoff AL, Joy SM, Hatzenbuehler JR, Saulsberry WJ, Coleman CI. AMSSM scientific statement concerning viscosupplementation injections for knee osteoarthritis: importance for individual patient outcomes. $\mathrm{Br} \mathrm{J}$ Sports Med. 2016;50(2):84-92.
43. Nelson AE, Allen KD, Golightly YM, Goode AP, Jordan JM. A systematic review of recommendations and guidelines for the management of osteoarthritis: the Chronic Osteoarthritis Management Initiative of the US Bone and Joint Initiative. Semin Arthritis Rheum. 2014;43(6):701-12. 